closed in 43 patients $(28.7 \%)$ with coil (28 in Nid-occlud, 10 in Cook, 5 in Gianturco coil), in 27 (18\%) patients with Amplatzer duct occluder (ADO) I, in 38 (25\%) patients with ADO II, in $24(16 \%)$ patients with ADO II Additional Size (ADO II AS) and in $18(12 \%)$ patients Occlutech Duct Occluder (ODO) device. All 6 patients with failed PDA closure were attempt to be closed with coil. The mean duct diameter was $2.1 \pm 1.24 \mathrm{~mm}$, and the mean $\mathrm{Qp} / \mathrm{Qs}$ ratio was $2 \pm$ 0.95. Ductus diameter was greater in ADO I and ODO group than coil and ADO IIAS group, and in ADO I group than ADO II group. The Qp/Qs ratio was higher in ADO I group compared to the coil, ADO II and ADO IIAS groups, in ODO group than coil and ADO IIAS groups and in ADO II group than coil group. In a patient with residual shunt after PDA closure, a second coil was placed in the ductus after 1 year. The early closure rate was $85.3 \%$ after the procedure and the complete closure rate at the first day echocardiography was $99.3 \%$. In the follow-up, mild left pulmonary artery stenosis in 2 patients and mild stenosis in the descending aorta in 3 patients were detected.

Conclusion Transcatheter closure of PDA in children is a treatment option that can be preferred safely depending on the shape of the duct, device selection and clinician's experience. With the increase of device diversity, the success rate of percutaneous closure is increasing. In our clinic, our successful closure rate increased with the introduction of duct occluder devices.

\section{GP24 SERUM RENALASE LEVELS IN ADOLESCENTS WITH PRIMARY HYPERTENSION}

${ }^{1}$ Marta Lemiesz, ${ }^{1}$ Edyta Tenderenda-Banasiuk*, ${ }^{1}$ Katarzyna Taranta-Janusz, ${ }^{2}$ Dorota Sosnowska, ${ }^{1}$ Anna Wasilewska. 'Department of Pediatrics and Nephrology, Medical University of Bialystok, Biahystok, Poland; ' ${ }^{2}$ epartment of Obstretrics- Gynecology, Medical Hospital in Garwolin, Garwolin, Poland

\subsection{6/archdischild-2019-epa.91}

Background The prevalence of hypertension continues to rise in pediatric populations. Recent studies suggest that renalase plays an important role in blood pressure regulation. The aim of this study was to evaluate serum renalase concentrations in hypertensive children.

Methods This study was a prospective cohort analysis of 88 adolescents (40 girls; 48 boys) aged 11 - 18 years, divided into two groups: HT - 38 subjects with primary hypertension and $\mathrm{R}$ - reference group - 50 subjects with normal blood pressure. Serum renalase concentration was measured using a commercial enzyme-linked immunosorbent assay kit (USCN Life Science Inc., China).

Results Hypertensive patients had higher serum renalase levels (median $29.8 \mu \mathrm{g} / \mathrm{mL}$; Q1-Q3: 26.1 - 35.8) than the reference group (median 26.8; Q1-Q3: 22.96 - 29.4, p<0.01). Serum renalase was strongly related to serum uric acid levels. In hypertensive patients, serum renalase was positively correlated with $24 \mathrm{H}-\mathrm{SBP}$ and $24 \mathrm{H}-\mathrm{DBP}$ and $24 \mathrm{H}-\mathrm{SBP}$ and $24 \mathrm{H}-\mathrm{DBP} \mathrm{Z}-$ score (LMS).

Conclusion Our results allow us to draw the conclusion that serum renalase is correlated with blood pressure elevation. Special attention should be drawn to the correlation between renalase and serum uric acid levels not only in hypertensive, but also in normotensive teenagers. Further studies are needed to answer the question if increased serum renalase may be a predisposing factor to hypertension in normotensive patients with hyperuricemia.

\section{REFERENCE}

1. Lemiesz M, Tenderenda-Banasiuk E, Sosnowska D, Taranta-Janusz K, Wasilewska A. Serum Renalase Levels in Adolescents with Primary Hypertension. Pediatr Cardiol. 2018 Aug;39(6):1258-1264. doi: 10.1007/s00246-018-1891-y. Epub 2018 May 10. PubMed PMID: 29748702; PubMed Central PMCID: PMC6096843.

\section{GP25 COMPARISON OF ASSOCIATIONS AND OUTCOMES OF PRENATALLY AND POSTNATALLY DIAGNOSED RIGHT AORTIC ARCH: SOUTH WALES' EXPERIENCE}

Ana Maria Irimia*, Amos Wong, Orhan Uzun. Noah's Ark Children's Hospital for Wales, Cardiff, UK

\subsection{6/archdischild-2019-epa.92}

Objectives To evaluate clinical associations and outcomes of antenatally and postnatally diagnosed Right Aortic Arch (RAA) in South Wales.

Methods Retrospective observational study of all RAA patients diagnosed in South Wales between 2000-2018.

Results We identified 38 cases of RAA, 68.4\% antenatally diagnosed and $31.6 \%$ postnatally. Following the introduction of a structured teaching programme of fetal 3 vessels trachea (3VT) views to sonographers in South Wales in 2013, the pick-up rate of antenatally diagnosed RAA has increased, from an average of 1 patient per year prior to 2013, to 9 patients in 2018 . $100 \%$ of cases were diagnosed antenatally starting from 2015. 57.7\% of antenatally diagnosed cases were referred specifically for RAA, and $7.7 \%$ for suspicion of abnormal arch, while $66.7 \%$ of postnatally diagnosed patients were referred for associated cardiac conditions. Two asymptomatic patients $(16.7 \%)$ from the postnatal group were referred for cardiac screening after being diagnosed with DiGeorge and 1 patient $(8.3 \%)$ for RAA symptoms.

Associated cardiac conditions were found in $65.8 \%$, while $32.4 \%$ had isolated RAA. The most common associated cardiac conditions were pulmonary stenosis or atresia and ventricular septal defect, at $23.7 \%$ each, followed by tetralogy of Fallot (18.4\%). 3 patients (7.9\%) had 22q11 microdeletion, all in the isolated group. One patient had Edwards syndrome and one patient had CHARGE syndrome $(2.6 \%)$ in non-isolated group. 7 patients $(18.4 \%)$ had non-cardiac conditions. The branching pattern was described in $34.2 \%$ of cases, of which 6 cases $(46.15 \%)$ being RAA with mirror image branching (MIB) and 4 cases $(30.76 \%)$ being RAA with aberrant left subclavian artery (ALSCA). 4 cases of MIB compared to 1 case of ALSCA had associated cardiac conditions.

$81.6 \%$ of patients with RAA were asymptomatic. 6 patients $(15.8 \%)$ had respiratory symptoms, of which 4 had isolated RAA, with a median age of onset of 2.2 months, and one patient $(2.6 \%)$ had apnoeic episodes (on prostaglandin for pulmonary atresia). One patient from the isolated group and one patient from the associated group underwent corrective surgery for vascular ring for significant symptoms. The majority of isolated RAA $(82.6 \%)$ were discharged.

Conclusions 3VT view resulted in improved antenatal diagnosis of RAA. Most RAA were associated with other cardiac conditions. RAA were mostly asymptomatic, with few developing respiratory symptoms, with a median onset at 2.2 months of age. 